DAMTP-2004-34

hep-th/0403281

\title{
Casimir effect and thermodynamics of horizon instabilities
}

\author{
Sean A. Hartnoll \\ s.a.hartnoll@damtp.cam.ac.uk \\ DAMTP, Centre for Mathematical Sciences, Cambridge University \\ Wilberforce Road, Cambridge CBз OWA, UK
}

\begin{abstract}
We propose a dual thermodynamic description of a classical instability of generalised black hole spacetimes. From a thermodynamic perspective, the instability is due to negative compressibility in regions where the Casimir pressure is large. The argument indicates how the correspondence between thermodynamic and classical instability for horizons may be extended to cases without translational invariance.
\end{abstract}




\section{Introduction}

The intriguing connection between classical and local thermodynamic instability $[1,2,3,4]$ has partially been behind a renewed interest in the Gregory-Laflamme instability of black branes $[5,6,7,8,9,10,11,12,13,14,15]$. The connection states that translationally invariant horizons are classically stable if and only if they are locally thermodynamically stable $[1,2,3]$. The objective of this work is to suggest a dual thermodynamic description of a recently discovered black hole instability. The novel aspects of the connection presented here are that the horizon is inhomogeneous and that the thermodynamic instability is due to a Casimir effect.

Local thermodynamic stability requires the Helmholtz free energy of the system to be a concave function of temperature and a convex function of volume. The known instabilities of black branes are due to a negative heat capacity, $C_{V}=-T \partial^{2} F /\left.\partial T^{2}\right|_{V}<0$. In contrast, the instability in this paper will be due to a negative isothermal compressibility $K_{T}^{-1}=$ $V \partial^{2} F /\left.\partial V^{2}\right|_{T}<0$.

We work within the context of the AdS/CFT correspondence [16, 17, 18]. Amongst other things, the AdS/CFT correspondence relates the physics of black holes in $\mathrm{AdS}_{d+2}$ to a thermal field theory living on the 'boundary' $S^{1} \times S^{d}[18,19]$. In the conclusion we note that it should be possible to extend the ideas presented here to general inhomogeneous horizons, not necessarily embedded in an AdS-like geometry.

Section 2 reviews an instability of generalised black holes [20, 21] with a negative cosmological constant [22]. We further recall how the criterion for classical instability of the black hole translates into dual field theory language.

Section 3 calculates the energy momentum tensor of the thermal field theory dual to the black hole spacetime using holographic renormalisation [23, 24, 25, 26, 27]. The thermal field theory has an ultrastatic background $S^{1} \times \mathcal{M}$, with $\mathcal{M}$ an arbitrary dimension $d$ Einstein manifold. There exists an ambiguity in the energy momentum tensor due to local curvature polynomials of mass dimension $(d+1) / 2$. Generically, these produce an inhomogeneous Casimir energy and pressure. We show how these terms may cause a thermodynamic instability. This thermodynamic instability appears to be a suitable dual description to the aforementioned classical instability of the black hole bulk spacetime.

Section 4 illustrates the previous considerations in the case when the horizon of the black hole is given by a Böhm metric on $S^{5}[28,21]$. The thermal field theory in this case has background $S^{1} \times S_{\text {Böhm }}^{5}$.

The last section is the discussion. The argument we have presented is not rigorous and 
we clarify what would be needed for a watertight discussion. Further applications of the Casimir effect to horizon instabilities are suggested.

\section{Instability of generalised AdS black holes}

We start by reviewing the stability of generalised black holes $[20,22]$ with a negative cosmological constant. Generalised black holes take the form

$$
d s^{2}=-f(r) d t^{2}+\frac{d r^{2}}{f(r)}+r^{2} d s_{d}^{2},
$$

where $f(r)=1-\left(1+r_{+}^{2} / L^{2}\right)\left(r_{+} / r\right)^{d-1}+r^{2} / L^{2}$. The event horizon is at $r=r_{+}$. If $d s_{d}^{2}$ were the round metric on $S^{d}$, then the spacetime would be the usual Schwarzschild black hole in AdS space. However, the $d+2$ dimensional vacuum Einstein equations allow the horizon metric $d s_{d}^{2}$ to be any $d$ dimensional Einstein metric on a Riemannian manifold $\mathcal{M}$

$$
R_{a b}=(d-1) g_{a b}
$$

The resulting spacetime is called a generalised black hole. The asymptotic geometry of the background has now changed and the dual thermal field theory lives on $S^{1} \times \mathcal{M}$.

The stability of generalised black holes has recently been investigated [22, 20, 21]. For large, $r_{+} / L \gg 1$, generalised AdS black holes one finds

$$
\lambda_{L}<-\frac{r_{+}^{2}}{L^{2}} \times \mathcal{O}(1) \quad \Leftrightarrow \quad \text { instability }
$$

where $\lambda_{L}$ is the minimum eigenvalue of the Lichnerowicz operator on $\mathcal{M}$. Recall that the Lichnerowicz operator acts on symmetric rank two tensors as

$$
\left(\Delta_{L} h\right)_{a b}=2 R_{a b d}^{c} h_{c}^{d}+R_{c a} h_{b}^{c}+R_{c b} h_{a}^{c}-\nabla^{c} \nabla_{c} h_{a b} .
$$

Note from (3) that the minimum Lichnerowicz eigenvalue needs to be very negative in order for instability to occur. Examples of Einstein manifolds with large negative Lichnerowicz eigenvalues are the Böhm metrics on $S^{5} \ldots S^{9}$ and on products of spheres [28, 21]. We will use these metrics as examples below.

The criterion may be translated into field theory language using the standard AdS/CFT dictionary to give [22]

$$
T^{2}<-\lambda_{L} \times \mathcal{O}(1) \Leftrightarrow \text { instability }
$$

where $T$ is the temperature of the field theory. Thus, the AdS/CFT correspondence predicts a critical temperature in the dual field theory, $T_{C}^{2} \sim\left|\lambda_{L}\right|$. 
The Lichnerowicz operator is not a particularly natural object to consider in field theories where gravity is nondynamical. On Einstein manifolds with positive curvature the minimum Lichnerowicz eigenvalue is related to the Weyl curvature. Roughly, one expects [21]

$$
\left.\lambda_{L}^{2} \sim C_{a b c d} C^{a b c d}\right|_{\max .} \sim \mathfrak{R}^{2}
$$

where we use $\mathfrak{R}$ to denote a typical magnitude of curvature of the geometry $\mathcal{M}$, and $\left.C_{a b c d} C^{a b c d}\right|_{\max }$ is the maximum value taken by $C_{a b c d} C^{a b c d}$ on the manifold. Putting these statements together, the critical temperature becomes

$$
T_{C}^{2} \sim \Re
$$

Note that the Ricci scalar, $R$, is fixed, so in the limit of large negative $\lambda_{L}$ we have $R \ll \Re$. This hierarchy is important for consistency because the large black hole limit $r_{+} / L \gg 1$ translates into the field theory condition $T \gg R$.

In the next section we present a potential field theory instability occurring at this critical temperature.

\section{Casimir instability of field theory thermodynamics}

\subsection{Holographic energy momentum tensor}

The thermodynamics of the dual field theory in the strong coupling regime may be studied using holographic renormalisation [23, 24, 25, 26, 27]. This is the regime dual to the weakly curved gravitational description in the bulk and is therefore the regime in which we expect to find a field theory instability corresponding to the black hole instability.

For concreteness we will work now with seven dimensional black holes and a six dimensional boundary. This allows the problem to be embedded into the $A d S_{7} \times S^{4}$ version of the AdS/CFT correspondence and will allow us in the next section to use five dimensional Böhm metrics as concrete examples. We will work from now on with a Euclidean signature, to describe finite temperature physics.

The expectation value of the energy momentum tensor of the dual theory is given by

$$
<T^{\mu \nu}>=\left.\frac{2}{\sqrt{\operatorname{det} g}} \frac{\partial S}{\partial g_{\mu \nu}}\right|_{\text {renormalised }},
$$

where $g_{\mu \nu}$ is the metric on the boundary of spacetime. The gravitational action, $S$, has three parts: the Einstein-Hilbert action, the Gibbons-Hawking boundary term and the counterterm action. The counterterm action is defined on the boundary of spacetime but 
differs crucially from the Gibbons-Hawking term in that it is a local functional of intrinsic boundary curvatures only. This implies that it does not affect the bulk equations of motion. The counterterms are added to cancel divergences in the action due to the diverging volume of the spacetime at infinity.

The calculation of the dual energy momentum tensor for a general spacetime with negative cosmological constant and a six dimensional boundary has been performed, for example, in [23]. It is straightforward to apply their formulae to the generalised black hole spacetime (1). Write the boundary metric as

$$
g_{\mu \nu} d x^{\mu} d x^{\nu}=d \tau^{2}+g_{a b} d x^{a} d x^{b}
$$

We have $\mu, \nu$ running over all the boundary coordinates and $a, b \ldots$ running over the spatial directions of the boundary. We find

$$
\begin{aligned}
& <T_{00}>=\frac{3 L^{5}}{8 \pi G_{7} r_{0}^{6}}\left[-\frac{5}{6} \frac{r_{+}^{4}}{L^{4}}\left(1+\frac{r_{+}^{2}}{L^{2}}\right)+\frac{5}{48}\right] g_{00}+\left.T_{00}\right|_{\text {f.c. }}, \\
& <T_{a b}>=\frac{3 L^{5}}{8 \pi G_{7} r_{0}^{6}}\left[\frac{1}{6} \frac{r_{+}^{4}}{L^{4}}\left(1+\frac{r_{+}^{2}}{L^{2}}\right)-\frac{1}{48}\right] g_{a b}+\left.T_{a b}\right|_{\text {f.c. }},
\end{aligned}
$$

where f.c. stands for 'finite counterterms', described below, and $r_{0}$ is an arbitrary scale introduced during the renormalisation. Dependence on $r_{0}$ will disappear shortly. The result (10) may be reexpressed in terms of field theory quantities by relating the radius of a large black hole, $r_{+} / L \gg 1$, to the field theory temperature, using the $A d S_{7} \times S^{4}$ relation between the AdS length and Newton's constant and introducing the Ricci scalar of $g_{a b}$ :

$$
T \approx \frac{3}{2 \pi} \frac{r_{+}}{r_{0} L}, \quad \frac{1}{G_{7}}=\frac{2^{4}}{3 \pi^{2}} \frac{N^{3}}{L^{5}}, \quad R=\frac{20}{r_{0}^{2}} .
$$

This gives

$$
\begin{aligned}
& <T_{00}>=-\frac{5 N^{3}}{3 \pi^{3}}\left[\left(\frac{2 \pi}{3}\right)^{6} T^{6}-\left(\frac{1}{5 \cdot 2^{3}}\right)^{3} R^{3}\right] g_{00}+\left.T_{00}\right|_{\text {f.c. }}, \\
& <T_{a b}>=\frac{N^{3}}{3 \pi^{3}}\left[\left(\frac{2 \pi}{3}\right)^{6} T^{6}-\left(\frac{1}{5 \cdot 2^{3}}\right)^{3} R^{3}\right] g_{a b}+\left.T_{a b}\right|_{\text {f.c. }} .
\end{aligned}
$$

The Ricci scalar term is in fact negligible because the large black hole limit implies $T^{2} \gg R$.

The origin of the finite counterterm contribution is as follows. The counterterm boundary action consists of curvature scalars. The terms in the action that are scalars of mass dimension less than six diverge as the boundary is taken to infinity and are used to cancel the divergences of the bulk gravitational action. Scalars of mass dimension greater than six are negligible as the boundary goes to infinity. Scalars of mass dimension precisely six remain finite. Thus, one is free to add these scalars to the countertem action. 
The same ambiguity also exists in the dual field theory. The coefficient of a mass dimension six curvature scalar in a six dimensional action is dimensionless and should therefore be viewed as a renormalisable coupling. The value of the coupling is thus generically not zero. Its value is not determined by the theory but needs to be observed 'experimentally'. The contribution of these terms to the action might be thought of as a position dependent cosmological constant. The variation of these terms with respect to the boundary metric gives the contribution to the energy momentum tensor that we have included above in (10) and (12). Due to the fact that there this constitutes a temperature independent contribution to the energy and pressure, we call the presence of these terms a Casimir effect.

\subsection{Thermodynamic instabilities - general argument}

Before considering explicitly the possible form of the finite counterterm contribution to the energy momentum tensor, we will see how such terms can produce thermodynamic instabilities. The following section offers a concrete example. Recall that we are interested in manifolds with a curvature scale much larger than the constant Ricci scalar $\mathfrak{R} \gg R$. The picture one has in mind is the following.

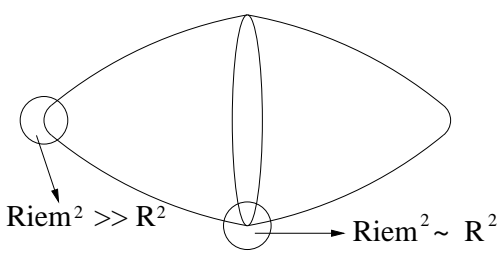

Figure 1: Manifold with two curvature scales, $\left.\operatorname{Riem}^{2}\right|_{\max } \sim \mathfrak{R}^{2} \gg R^{2}$.

In the region where the curvature scale $\mathfrak{R}$ is large, $\mathfrak{R} \gg R$, the pressure in (12) will be

$$
P \sim N^{3}\left[T^{6} \pm \mathfrak{R}^{3}\right]
$$

We are neglecting positive order one coefficients. We have taken the $N^{3}$ term as an overall factor because one would expect the Casimir contribution to be proportional to the number of degrees of freedom.

If the temperature is sufficiently high, $T^{2} \gg \mathfrak{R}$, then the field theory thermodynamics is just that of black body radiation of $\mathcal{O}\left(N^{3}\right)$ species. However, if $T^{2}<\mathfrak{R}$, then the Casimir pressure is dominant in the regions of large curvature. This is consistent with the large temperature approximation we are making, $T^{2} \gg R$, because of the hierarchy of curvatures 
$\Re \gg R$. Thus, the qualitative thermodynamic behaviour in the strong curvature regions appears to change at around $T_{C}^{2} \sim \Re$.

The curvature $\mathfrak{R}$ has an associated lengthscale $L_{\mathfrak{R}}=\mathfrak{R}^{-1 / 2}$. This lengthscale gives the size of the strong curvature region. The lengthscale associated with the wavelength of thermal excitations is $L_{T}=T^{-1}$. In the lower temperature regime $T^{2}<T_{C}^{2} \sim \mathfrak{R}$, the thermal wavelength is larger than the size of the strong curvature region: $L_{T}>L_{\mathfrak{R}}$. Therefore the bulk of the thermal spectrum is not excited in this region. Effectively, the region is not in thermodynamic equilibrium with the rest of the radiation. To first approximation, the strongly curved region is at zero temperature. In this case, let us consider the behaviour of the strong curvature region as an independent system. The volume of this region is

$$
V \sim L_{\mathfrak{R}}^{5}=\left(\frac{1}{\Re}\right)^{5 / 2} .
$$

Writing the pressure (13) in terms of the volume of this region

$$
P \sim \pm\left(\frac{1}{V}\right)^{6 / 5}
$$

Consider the isothermal compressibility

$$
K_{T}^{-1}=-V\left(\frac{\partial P}{\partial V}\right)_{T}= \pm \frac{6}{5}\left(\frac{1}{V}\right)^{6 / 5}
$$

The compressibility may also be expressed in terms of the Helmholtz free energy by using the Maxwell relation $P=-\partial F /\left.\partial V\right|_{T}$

$$
K_{T}^{-1}=V\left(\frac{\partial^{2} F}{\partial V^{2}}\right)_{T} .
$$

Thus where the compressibility is negative, the free energy is not a convex function of the volume and the system is thermodynamically unstable.

We see that there will be a thermodynamic instability at $T^{2}=T_{C}^{2} \sim \mathfrak{R}$ if the sign of the Casimir pressure in (13) is negative. We will see below that in practice the contributions to the pressure come with both positive and negative signs. The present argument indicates the generic possibility of instability, rather than a necessary instability. The localisation of the instability to the strong curvature regions is mirrored by the classical black hole instability, where the unstable mode is concentrated in these regions [21].

The dual operator to the unstable mode [22] is a linear combination of spatial components of the energy momentum tensor. Below the critical temperature one expects the vacuum expectation value of this operator, which are components of the pressure, to shift. 
This suggests that there is a new stable phase where the curvature does not give the dominant contribution to the pressure.

This instability should be compared with previous matchings between dynamical and thermodynamic instability $[1,2,3,4]$. The instability in these works is due to a negative heat capacity

$$
C_{V}=T\left(\frac{\partial S}{\partial T}\right)_{V}=-T\left(\frac{\partial^{2} F}{\partial T^{2}}\right)_{V}
$$

\subsection{Contributions to the Casimir pressure}

We can be more precise about the curvature terms contributing to the energy-momentum tensor in the strongly curved regions. In six spacetime dimensions, there are 17 curvature scalars that have mass dimension six [29]. These will generically be present in the action and their variation will contribute to the energy momentum tensor. However, not all of the contributions will be large. The spatial background is Einstein, and consequently has constant Ricci scalar, $R$, and Ricci tensor. Some of the tensors will be $\mathcal{O}\left(R^{3}\right)$ or $\mathcal{O}\left(R^{2} \mathfrak{R}\right)$ or $\mathcal{O}\left(R \Re^{2}\right)$ instead of $\mathcal{O}\left(\mathfrak{R}^{3}\right)$. These will be negligible in the region where $\mathfrak{R} \gg R$.

Given that the field theory does not specify the coefficients of the curvature terms in the action, there is not much to be gained at this stage from starting with the action. Instead of calculating the variation of the 17 curvature scalars, we will start by directly considering all possible rank two tensors that have mass dimension six. Although there are many such tensors [29], if we consider a background of the form $S^{1} \times \mathcal{M}$, with $\mathcal{M}$ Einstein, then there are only eight independent rank two tensors with mass dimension six that are $\mathcal{O}\left(\mathfrak{R}^{3}\right)$. Explicitly,

$$
\left.T_{\mu \nu}\right|_{\text {f.c. }}=\sum_{i=1}^{8} k_{i} K_{\mu \nu}^{(i)}+\mathcal{O}\left(R \mathfrak{R}^{2}\right),
$$

for some coefficients $k_{i}$ and where the tensors $K_{\mu \nu}^{(i)}$ are $^{1}$

$$
\begin{aligned}
K_{\mu \nu}^{(1) \cdots(8)}= & \left\{R^{p q r s} \nabla_{(\mu} \nabla_{\nu)} R_{p q r s}, \nabla_{\mu} R^{p q r s} \nabla_{\nu} R_{p q r s}, \nabla_{s} R^{p q r}{ }_{\mu} \nabla^{s} R_{p q r \nu}, R^{p q r s} R_{p q}{ }^{t} R_{r s t \nu},\right. \\
& \left.R^{p q r s} R_{p r \mu}{ }^{t} R_{q t s \nu}, R^{p q r s} R_{p q r}{ }^{t} R_{s \mu t \nu}, \nabla_{t} R^{\text {pqrs }} \nabla^{t} R_{p q r s} g_{\mu \nu}, R^{p q r s} R_{p}{ }^{t}{ }^{u} R_{q t s u} g_{\mu \nu}\right\} .
\end{aligned}
$$

All other linearly independent rank two tensors with mass dimension six either vanish or contain factors of the Ricci scalar when considered in an Einstein background. This may be worked out from the basis of tensors given in [29]. The $S^{1}$ factor in the metric does not

\footnotetext{
${ }^{1}$ For a general six dimensional metric there is a ninth independent tensor $R^{p q r s} R_{p q t u} R_{r s}{ }^{t u} g_{\mu \nu}$, but this is not independent in five dimensions, or in the case $S^{1} \times \mathcal{M}$. Thus in our backgrounds it is not $\mathcal{O}\left(\mathfrak{R}^{3}\right)$.
} 
contribute to any of the curvature tensors and so all the summations involving curvature tensors are over the five spatial directions - compare with the notation of equation (9).

Not all of these terms are obtainable from varying an action. We must impose two constraints. Firstly, diffeomorphism invariance of the action implies that the energy momentum tensor is transverse (the Noether constraint) because under the diffeomorphism $\delta g_{\mu \nu}=\nabla_{(\mu} \xi_{\nu)}$

$$
0=\delta_{\xi} S=\int \frac{\delta S}{\delta g_{\mu \nu}} \nabla_{(\mu} \xi_{\nu)} d \Omega=-\int\left(\nabla_{\mu} \frac{\delta S}{\delta g_{\mu \nu}}\right) \xi_{\nu} d \Omega \quad \Rightarrow \quad \nabla_{\mu} \frac{\delta S}{\delta g_{\mu \nu}}=\nabla_{\mu} T^{\mu \nu}=0
$$

Secondly, in six dimensions an action given by scalars with mass dimension six is invariant under constant Weyl rescalings. This implies that the trace of the corresponding energy momentum tensor must be a total derivative. Under $\delta g_{\mu \nu}=\sigma g_{\mu \nu}$ :

$$
\int \frac{\delta S}{\delta g_{\mu \nu}} \sigma g_{\mu \nu} d \Omega=\delta_{\sigma} S=\int \nabla_{\mu} \sigma J^{\mu} d \Omega=-\int \sigma \nabla_{\mu} J^{\mu} d \Omega \quad \Rightarrow \quad g_{\mu \nu} \frac{\delta S}{\delta g_{\mu \nu}}=T=-\nabla_{\mu} J^{\mu},
$$

for some vector $J^{\mu}$. This total derivative contributes to the non-universal part of the Weyl anomaly $[30,31]$.

The trace constraint is the easier to apply. The possible candidates for $J^{\mu}$ are rank one tensors with mass dimension five or indeed zero. The only relevant nonzero possibility is $J_{\mu}=R^{p q r s} \nabla_{\mu} R_{\text {pqrs }}$ [29]. Considering the eight tensors in (20) it follows that there are seven admissible combinations

$$
\left\{K_{\mu \nu}^{(1)}+K_{\mu \nu}^{(2)}, K_{\mu \nu}^{(1)}+K_{\mu \nu}^{(3)}, K_{\mu \nu}^{(1)}+\frac{1}{6} K_{\mu \nu}^{(7)}, K_{\mu \nu}^{(5)}-\frac{1}{6} K_{\mu \nu}^{(8)}, K_{\mu \nu}^{(6)}, K_{\mu \nu}^{(4)}, K_{\mu \nu}^{(1)}+4 K_{\mu \nu}^{(5)}\right\}
$$

where, in taking the trace, any terms arising that are not $\mathcal{O}\left(\mathfrak{R}^{3}\right)$ are ignored. It is assumed that any contributions to the trace smaller than $\mathcal{O}\left(\mathfrak{R}^{3}\right)$ may be cancelled using other small terms. The footnote of the previous page is important for this calculation.

Now consider the divergences of these seven tensors. We need combinations of the tensors in (23) that have vanishing divergence. The appendix indicates how one goes about finding the combinations systematically. The result is four linearly independent tensors

$$
\begin{aligned}
H_{\mu \nu}^{(1)} & =K_{\mu \nu}^{(4)}-2 K_{\mu \nu}^{(5)}-K_{\mu \nu}^{(6)}+\frac{1}{3} K_{\mu \nu}^{(8)}, \\
H_{\mu \nu}^{(2)} & =-K_{\mu \nu}^{(1)}+K_{\mu \nu}^{(2)}-16 K_{\mu \nu}^{(5)}+\frac{4}{3} K_{\mu \nu}^{(8)}, \\
H_{\mu \nu}^{(3)} & =-\frac{1}{2} K_{\mu \nu}^{(1)}+K_{\mu \nu}^{(3)}-10 K_{\mu \nu}^{(5)}+\frac{2}{3} K_{\mu \nu}^{(8)}, \\
H_{\mu \nu}^{(4)} & =-K_{\mu \nu}^{(1)}-8 K_{\mu \nu}^{(5)}+\frac{1}{2} K_{\mu \nu}^{(7)}-\frac{4}{3} K_{\mu \nu}^{(8)} .
\end{aligned}
$$


Let us summarise what we have just achieved. We wanted to calculate possible contributions to the energy momentum tensor coming from a mass dimension six curvature scalar in the action. Further, we were only interested in terms that are $\mathcal{O}\left(\mathfrak{R}^{3}\right)$ and hence give the dominant contribution in the strongly curved regions. These contributions had to be rank two tensors with mass dimension six. We have shown that the only tensors satisfying all these conditions are the four in equation (24). We may now calculate these terms in a concrete background.

\section{Dimension six tensors of Böhm metrics on $S^{5}$}

The Böhm metrics [28] serve as an illustration of the ideas we have introduced. We will consider Böhm metrics on $S^{5}$. The metrics have the form

$$
d s_{5}^{2}=d \theta^{2}+a(\theta)^{2} d \Omega_{2}^{2}+b(\theta)^{2} d \widetilde{\Omega}_{2}^{2}
$$

where $d \Omega_{2}^{2}$ and $d \widetilde{\Omega}_{2}^{2}$ are round metrics on spheres $S^{2}$ and $\widetilde{S}^{2}$, with polar coordinates $(\alpha, \beta)$ and $(\tilde{\alpha}, \tilde{\beta})$ respectively. The coordinate $\theta$ has range 0 to $\theta_{\mathrm{f}}$. The Einstein equations imply some nonlinear differential equations for $a(\theta)$ and $b(\theta)$. The boundary conditions for topology $S^{5}$ are that $a(0)=0, b(0)=b_{0}$ and $a\left(\theta_{\mathrm{f}}\right)=a_{0}, b\left(\theta_{\mathrm{f}}\right)=0$. In fact, the solutions to the equations on $S^{5}$ have $a(\theta)=b\left(\theta_{\mathrm{f}}-\theta\right)$. There is a discrete infinity of solutions, at specific values of $b_{0}$. Solutions exist for arbitrarily small $b_{0}$. As $b_{0} \rightarrow 0$ the metric develops conical singularities at $\theta=0, \theta=\theta_{\mathrm{f}}$. Thus $b_{0}$ gives the resolution of the singularity. We expect to find $\mathfrak{R} \sim b_{0}^{-2}$.

We can evaluate the four contributions to the energy momentum tensor of (24) at the points $\theta=0$ and $\theta=\theta_{\mathrm{f}}$ using Taylor series expansions for the functions $a(\theta)$ and $b(\theta)[21]$. The results for $b_{0} \ll 1$ are contained in Table 1 below. One may obtain the values at $\theta=\theta_{\mathrm{f}}$ by letting $H_{\alpha}^{(i)_{\alpha}} \leftrightarrow H_{\tilde{\alpha}}^{(i) \tilde{\alpha}}, H_{\beta}^{(i)_{\beta}} \leftrightarrow H_{\tilde{\beta}}^{(i) \tilde{\beta}}$ and $H_{\theta}^{(i)_{\theta}}$ remains the same.

We are working in units where the Ricci scalar $R=20$, so the condition $b_{0} \ll 1$ is the introduction of a new scale: $b_{0}^{-2} \sim \mathfrak{R} \gg R$. The first observation we can make about Table 1 is that the contribution to the pressure is $\mathcal{O}\left(\mathfrak{R}^{3}\right)$ as expected. The pressure in each direction is given by $P_{x}^{(i)}=k_{i} H_{x}^{(i)}$. The second observation is that contributions to the pressure from $H_{\mu \nu}^{(2)}$ have different signs for different directions. Thus, whichever the sign of the coefficient, $k_{2}$, of $H_{\mu \nu}^{(2)}$ in the energy momentum tensor, one of these pressures will be negative, contributing towards a thermodynamic instability as outlined above. Of course, all the pressures could still be made positive by adding a larger positive contribution from one of the other tensors. 


\begin{tabular}{|c||c|}
\hline Tensor components & At $\theta=0$ \\
\hline \hline$H_{\theta}^{(1) \theta}=H_{\alpha}^{(1) \alpha}=H_{\beta}^{(1) \beta}=H_{\tilde{\alpha}}^{(1) \tilde{\alpha}}=H_{\tilde{\beta}}^{(1) \tilde{\beta}}$ & $\frac{32}{27 b_{0}^{6}}$ \\
\hline$\left\{H_{\theta}^{(2) \theta}, H_{\alpha}^{(2) \alpha}=H_{\beta}^{(2) \beta}, H_{\tilde{\alpha}}^{(2) \tilde{\alpha}}=H_{\tilde{\beta}}^{(2) \tilde{\beta}}\right\}$ & $\left\{\frac{64}{27 b_{0}^{6}},-\frac{128}{27 b_{0}^{6}},-\frac{256}{27 b_{0}^{6}}\right\}$ \\
\hline$\left\{H_{\theta}^{(3) \theta}, H_{\alpha}^{(3) \alpha}=H_{\beta}^{(3) \beta}, H_{\tilde{\alpha}}^{(3) \tilde{\alpha}}=H_{\tilde{\beta}}^{(3) \tilde{\beta}}\right\}$ & $\left\{\mathcal{O}\left(\frac{1}{b_{0}^{4}}\right),-\frac{32}{9 b_{0}^{6}},-\frac{176}{27 b_{0}^{6}}\right\}$ \\
\hline$\left\{H_{\theta}^{(4) \theta}, H_{\alpha}^{(4) \alpha}=H_{\beta}^{(4) \beta}, H_{\tilde{\alpha}}^{(4) \tilde{\alpha}}=H_{\tilde{\beta}}^{(4) \tilde{\beta}}\right\}$ & $\left\{-\frac{64}{27 b_{0}^{6}},-\frac{256}{27 b_{0}^{6}},-\frac{320}{27 b_{0}^{6}}\right\}$ \\
\hline
\end{tabular}

Table 1: Contributions to the pressure at $\theta=0$ for Böhm backgrounds. $P_{x}^{(i)}=k_{i} H_{x}^{(i) x}$.

\section{Summary, discussion and open questions}

Large generalised black holes with a negative cosmological constant tend to have dynamical instabilities if the horizon has a curvature scale which in some region is large compared to the constant Ricci scalar of the horizon, $\mathfrak{R} \gg R$. This predicts a critical temperature in the dual field theory: $T_{C}^{2} \sim \Re$.

We specialised to the case of a six dimensional field theory, as this corresponds to the lowest dimension in which unstable black holes are known and further may be considered within the $A d S_{7} \times S^{4}$ version of the AdS/CFT duality.

We have argued that field theories on curved backgrounds with a curvature scale $\Re \gg R$ can generically have a thermodynamic instability at $T_{C}^{2} \sim \mathfrak{R}$ due to the strong curvature region having negative compressibility. The instability is caused by contributions to the pressure from mass dimension six curvature tensors. These come from mass dimension curvature six scalars that are renormalisable vacuum terms in the field theory action. We calculated the explicit form of the contribution to the energy momentum tensor, and evaluated these tensors for the case of Böhm backgrounds.

There are various unsatisfactory aspects of our treatment. We do not have a precise matching. Whilst a precise criterion for black hole instability is known [22], this is not the case in field theory. This is because the coefficients of the curvature terms in the energy momentum tensor are not predicted by the field theory, but are rather empirically determined, just like coupling constants. Yet, the instability itself depends on the total contribution to the pressure having a specific sign. 
This situation potentially raises a conceptual problem for the AdS/CFT correspondence. The field theory has undetermined coefficients that correspond to finite terms in the boundary action on the gravitational side of the duality. However, these terms do not influence the dynamics of the bulk, and the classical stability of the bulk in particular. A possible resolution of the problem is that the bulk spacetime determines a specific renormalisation scheme for the field theory terms in question. In order for the matching of instabilities to be made precise, this renormalisation scheme would have to be known explicitly.

Nonetheless, the qualitative agreement is suggestive. It fits well into the recent discovery of agreement between thermodynamic and classical instabilities of horizons, and extends the connection to cases without translational invariance. An exciting possibility is that the ideas presented here could be adapted for use in other contexts where the stability of inhomogeneous horizons is of interest. In more general cases the Casimir energy would presumably not be that of a dual field theory, but rather due to gravitational degrees of freedom. A possible application of current interest is the stability of nonuniform black branes $[8,9,10,11]$. Can the stability be studied from a thermodynamic perspective? Can one associate a Casimir energy and pressure with general inhomogeneous horizons?

\section{Acknowledgments}

During this work I've had helpful comments from Ian Drummond, Gary Gibbons, Hugh Osborne, Ron Horgan, Rob Myers and Kostas Skenderis. I'd also like to thank David Berman, Neil Constable, Dumitru Ghilancea, Robert Helling, Giusseppe Policastro and Christian Stahn for some interesting conversations. This work is financially supported by the Sims scholarship.

\section{A Divergences of mass dimension six two-tensors}

This appendix contains formulae that are necessary for finding the independent linear combinations of the tensors in (23) that have vanishing divergence. This is done by expressing the divergences of each rank two tensor in terms of independent rank one tensors. The independent rank one tensors will be

$$
\begin{aligned}
& A_{\nu}^{(1)}=\nabla_{a} R_{\text {pqrs }} \nabla_{\nu} \nabla^{a} R^{p q r s} \\
& A_{\nu}^{(2)}=R_{\text {pqrs }} R_{e}{ }^{q}{ }_{a \nu} \nabla^{a} R^{\text {pers }} \\
& A_{\nu}^{(3)}=R_{\text {pqrs }} R_{e}{ }^{q}{ }_{a}{ }^{r} \nabla_{\nu} R^{\text {epas }}
\end{aligned}
$$




$$
A_{\nu}^{(4)}=R_{p q r s} R_{e a}^{q}{ }_{\nu} \nabla^{a} R^{p e r s}
$$

With a little work, one may then show that

$$
\left.\begin{array}{rl}
\nabla^{\mu} K_{\mu \nu}^{(1)} & =A_{\nu}^{(1)}-4 A_{\nu}^{(2)}+8 A_{\nu}^{(3)} \\
\nabla^{\mu} K_{\mu \nu}^{(2)} & =A_{\nu}^{(1)}+4 A_{\nu}^{(2)}+4 A_{\nu}^{(3)} \\
\nabla^{\mu} K_{\mu \nu}^{(3)} & =\frac{1}{2} A_{\nu}^{(1)}+3 A_{\nu}^{(2)}+A_{\nu}^{(3)} \\
\nabla^{\mu} K_{\mu \nu}^{(4)} & =2 A_{\nu}^{(4)} \\
\nabla^{\mu} K_{\mu \nu}^{(5)} & =\frac{1}{2} A_{\nu}^{(2)}-\frac{1}{2} A_{\nu}^{(3)} \\
\nabla^{\mu} K_{\mu \nu}^{(6)} & =-A_{\nu}^{(2)}+2 A_{\nu}^{(4)} \\
\nabla^{\mu} K_{\mu \nu}^{(7)} & =2 A_{\nu}^{(1)} \\
\nabla^{\mu} K_{\mu \nu}^{(8)} & =-3 A_{\nu}^{(3)}
\end{array}\right\}+\mathcal{O}\left(R \mathfrak{R}^{5 / 2}\right)
$$

where the $\mathcal{O}\left(R \Re^{5 / 2}\right)$ indicates that throughout the calculation, all Ricci tensors have been dropped. The results are exact if $R_{\mu \nu}=0$. Recall that we do this because we are interested in the dominant contributions in the region where $\mathfrak{R} \gg R$.

There is a fifth tensor that appears in these calculations which one might think is linearly independent

$$
A_{\nu}^{(5)}=R_{p q r s} R_{e{ }^{s}{ }_{a}} \nabla_{\nu} R^{p q a e} .
$$

However, this can be seen to be a multiple of $\nabla_{\nu}\left(R^{p q r s} R_{p q t u} R_{r s}{ }^{t u}\right)$. As we noted above, for the backgrounds we are considering, $R^{p q r s} R_{p q t u} R_{r s}{ }^{t u}$ is not $\mathcal{O}\left(\Re^{3}\right)$. We are only keeping track of leading order terms, so this term is not linearly independent.

Given these expressions for the divergences in terms of linearly independent one-tensors (27), it is simple linear algebra to find the two divergence free tensors of equation (24).

\section{References}

[1] S. S. Gubser and I. Mitra, "Instability of charged black holes in anti-de Sitter space," arXiv:hep-th/0009126.

[2] S. S. Gubser and I. Mitra, "The evolution of unstable black holes in anti-de Sitter space," JHEP 0108 (2001) 018 [arXiv:hep-th/0011127].

[3] H. S. Reall, "Classical and thermodynamic stability of black branes," Phys. Rev. D 64 (2001) 044005 [arXiv:hep-th/0104071].

[4] J. P. Gregory and S. F. Ross, "Stability and the negative mode for Schwarzschild in a finite cavity," Phys. Rev. D 64 (2001) 124006 [arXiv:hep-th/0106220]. 
[5] R. Gregory and R. Laflamme, "The Instability of charged black strings and p-branes," Nucl. Phys. B 428 (1994) 399 [arXiv:hep-th/9404071].

[6] R. Gregory and R. Laflamme, "Black Strings And P-Branes Are Unstable," Phys. Rev. Lett. 70 (1993) 2837 [arXiv:hep-th/9301052].

[7] G. T. Horowitz and K. Maeda, "Fate of the black string instability," Phys. Rev. Lett. 87 (2001) 131301 [arXiv:hep-th/0105111].

[8] S. S. Gubser, "On non-uniform black branes," Class. Quant. Grav. 19 (2002) 4825 [arXiv:hep-th/0110193].

[9] B. Kol, "Topology change in general relativity and the black-hole black-string transition," arXiv:hep-th/0206220.

[10] T. Wiseman, "From black strings to black holes," Class. Quant. Grav. 20 (2003) 1177 [arXiv:hep-th/0211028].

[11] T. Wiseman, "Static axisymmetric vacuum solutions and non-uniform black strings," Class. Quant. Grav. 20 (2003) 1137 [arXiv:hep-th/0209051].

[12] B. Kol and T. Wiseman, "Evidence that highly non-uniform black strings have a conical waist," Class. Quant. Grav. 20 (2003) 3493 [arXiv:hep-th/0304070].

[13] S. S. Gubser and A. Ozakin, "Universality classes for horizon instabilities," JHEP 0305 (2003) 010 [arXiv:hep-th/0301002].

[14] T. Hirayama and G. Kang, "Stable black strings in anti-de Sitter space," Phys. Rev. D 64 (2001) 064010 [arXiv:hep-th/0104213].

[15] G. Kang and J. Lee, "Classical stability of black D3-branes," JHEP 0403 (2004) 039 [arXiv:hep-th/0401225].

[16] J. M. Maldacena, "The large N limit of superconformal field theories and supergravity," Adv. Theor. Math. Phys. 2 (1998) 231 [Int. J. Theor. Phys. 38 (1999) 1113] [arXiv:hepth/9711200].

[17] S. S. Gubser, I. R. Klebanov and A. M. Polyakov, "Gauge theory correlators from non-critical string theory," Phys. Lett. B 428 (1998) 105 [arXiv:hep-th/9802109].

[18] E. Witten, "Anti-de Sitter space and holography," Adv. Theor. Math. Phys. 2 (1998) 253 [arXiv:hep-th/9802150]. 
[19] E. Witten, "Anti-de Sitter space, thermal phase transition, and confinement in gauge theories," Adv. Theor. Math. Phys. 2 (1998) 505 [arXiv:hep-th/9803131].

[20] G. Gibbons and S. A. Hartnoll, "A gravitational instability in higher dimensions," Phys. Rev. D 66 (2002) 064024 [arXiv:hep-th/0206202].

[21] G. W. Gibbons, S. A. Hartnoll and C. N. Pope, "Bohm and Einstein-Sasaki metrics, black holes and cosmological event horizons," Phys. Rev. D 67 (2003) 084024 [arXiv:hep-th/0208031].

[22] S. A. Hartnoll, "Instability of generalised AdS black holes and thermal field theory," JHEP 0308 (2003) 019 [arXiv:hep-th/0305001].

[23] S. de Haro, S. N. Solodukhin and K. Skenderis, "Holographic reconstruction of spacetime and renormalization in the AdS/CFT correspondence," Commun. Math. Phys. 217 (2001) 595 [arXiv:hep-th/0002230].

[24] V. Balasubramanian and P. Kraus, "A stress tensor for anti-de Sitter gravity," Commun. Math. Phys. 208 (1999) 413 [arXiv:hep-th/9902121].

[25] P. Kraus, F. Larsen and R. Siebelink, "The gravitational action in asymptotically AdS and flat spacetimes," Nucl. Phys. B 563 (1999) 259 [arXiv:hep-th/9906127].

[26] K. Skenderis, "Asymptotically anti-de Sitter spacetimes and their stress energy tensor," Int. J. Mod. Phys. A 16 (2001) 740 [arXiv:hep-th/0010138].

[27] R. C. Myers, "Stress tensors and Casimir energies in the AdS/CFT correspondence," Phys. Rev. D 60 (1999) 046002 [arXiv:hep-th/9903203].

[28] C. Böhm, "Inhomogeneous Einstein metrics on low-dimensional spheres and other lowdimensional spaces", Invent. Math. 134 (1998) 145.

[29] S. A. Fulling, R. C. King, B. G. Wybourne and C. J. Cummins, "Normal Forms For Tensor Polynomials. 1: The Riemann Tensor," Class. Quant. Grav. 9 (1992) 1151.

[30] F. Bastianelli, G. Cuoghi and L. Nocetti, "Consistency conditions and trace anomalies in six dimensions," Class. Quant. Grav. 18 (2001) 793 [arXiv:hep-th/0007222].

[31] S. Deser and A. Schwimmer, "Geometric Classification Of Conformal Anomalies In Arbitrary Dimensions," Phys. Lett. B 309 (1993) 279 [arXiv:hep-th/9302047]. 